\title{
Assemblies of conjugated polymers. Intermolecular and intramolecular effects on the photophysical properties of conjugated polymers*
}

\author{
Jinsang Kim \\ Division of Chemistry and Chemical Engineering, California Institute of Technology, \\ Pasadena, CA 91125, USA
}

\begin{abstract}
Conjugated polymers are emerging materials for electronic applications due to the tunability of their properties through variation of their chemical structure. Their applications, which currently include light-emitting diodes (LEDs), field effect transistors (FETs), plastic lasers, batteries, and sensors, are expanding to many new areas. The two critical parameters that determine the function of conjugated polymer-based devices are chemical structure and nanostructure of a conjugated polymer in the solid state. While the physical properties of isolated polymers are primarily controlled by their chemical structure, these properties are drastically altered in the solid state due to electronic coupling between polymer chains as determined by their interpolymer packing and conformation. However, the development of effective and precise methods for controlling the nanostructure of polymers in the solid state has been limited because polymers often fail to assemble into organized structures due to their amorphous character and large molecular weight.

In this review, recent developments of organizing methods of conjugated polymers and the conformation and interpolymer interaction effects on the photophysical properties of conjugated polymers are summarized.
\end{abstract}

\section{ASSEMBLY METHODS OF CONJUGATED POLYMERS}

Structural control of polymers in the solid state is a challenging task. The amorphous character combined with the large molecular weight prevent polymers from organizing into a controlled structure by conventional film preparation methods such as the spin-cast and drop-cast methods. The organization of conventional polymers has been achieved by special methods such as self-assembly, the Langmuir-Blodgett (LB) method, and order-disorder transition of block copolymers. These methods are now being exploited to organize conjugated polymers in the solid state.

\section{Layer-by-layer self-assembly}

The layer-by-layer (LBL) self-assembly method utilizes the electrostatic interactions between oppositely charged molecules [1]. Sequential dipping of a pretreated substrate into aqueous solutions containing oppositely charged polyelectrolytes produces a well-defined multilayer polymer film composed of alternatingly adsorbed oppositely charged polyelectrolytes.

*Pure Appl. Chem. 74, 2021-2081 (2002). A collection of invited, peer-reviewed articles by the winners of the 2002 IUPAC Prize for Young Chemists. 
Rubner and coworkers have systematically studied the feasibility of employing the LBL method for the assembly of conjugated polymers. Conjugated polyions such as positively charged poly( $p$-phenylenevinylene) (PPV) precursor, sulfonated polyaniline, and poly(thiophene-3-acetic acid) were first examined. In conjunction with oppositely charged conjugated or nonconjugated polyions, these conjugated polyions were successfully fabricated into alternating multilayer films. Parameters such as solution concentration, ionic strength of solution, and $\mathrm{pH}$ that can modify the film morphology by influencing charge interactions were elaborated [2,3]. Besides this chemical modification to introduce charged groups, the delocalized positive charges of p-type doped polypyrrole, polyaniline, and polyalkylthiophene were directly utilized to make assembled multilayers in combination with negatively charged polyanions [4-6]. Hydrogen bonding was also employed to fabricate polyaniline multilayer films. The amine or imine sites of a polyaniline backbone are known to form strong hydrogen bonds, enabling the LBL process in conjunction with aqueous solutions of nonionic polymers that form hydrogen bonds with polyanilines [7].

Other researchers have used the LBL self-assembly method to build multilayers of PPV, one of the most widely used conjugated polymers. A precursor approach was first examined. Positively charged PPV precursors and negatively charged polyelectrolytes were alternatingly assembled into the multilayer films. Subsequent heat treatment on the resulting multilayers under vacuum converted the precursor layers into fully conjugated PPV layers [8-12]. Alternatively, charge groups can be incorporated into the polymer backbone through side-chain modification. For example, attaching sulfonatedalkoxy side-groups into the PPV (MPS-PPV) endowed solubility in water, allowing alternating adsorption with cationic dendrimers to fabricate multilayer films [13,14]. Studies of a water-soluble poly( $p$-phenyleneethynylene) (PPE) with cationic side-chains also demonstrated that the LBL method is a good strategy to make layered structural conjugated polymer films by forming a stable alternating multilayer film with sulfonated polystyrene [15].

The LBL method is an effective method to obtain layered structural conjugated polymer films with controlled thickness. Morphology of polymer films can be controlled by manipulating electrostatic forces among charged side-chains through controlling solution concentration, ionic strength, and $\mathrm{pH}$ of an aqueous polymer solution. However, the LBL method has a practical limitation in controlling interpolymer orientation or alignment of polymers in a given layer. In this context, the LB technique may provide better control of the orientation and/or alignment of conjugated polymers.

\section{Langmuir-Blodgett technique}

The LB technique requires either amphiphilic polymers or hairy-rod polymers that are decorated with long alkyl side-chains to arrange polymers at the air-water interface [16]. Polythiophenes have been primarily explored for this assembly method. The intrinsic solubility problem has been tackled by introducing long alkyl groups at the 3-position of the thiophene rings. Attempts to assemble pure poly(alkylthiophenes) generally failed because the polymers do not form a stable monolayer at the air-water interface due to the formation of aggregates in spite of the hairy decoration $[17,18]$. The traditional hairy-rod polymers have macroscopically cylindrical shape on which the hairy long alkyl chains are evenly distributed. Therefore, the liquid-like alkyl chains effectively disperse the polymers at the air-water interface. However, poly(alkylthiophenes) have hairy-decorated board-like structure rather than a cylindrical rod. Hence, the long alkyl side-chains at the edge of the hairy board cannot effectively hinder the strong face-to-face $\pi$-interaction between thiophene backbones, allowing aggregate formation at the air-water interface. To prevent this aggregation phenomena, long-chain fatty acids such as stearic acid have been mixed with poly(alkylthiophenes), successfully generating a stable monolayer [17-24]. However, these fatty acids can alter the packing of polythiophenes in the transferred LB films, resulting in deterioration of the physical properties of the films. Efforts have been made to prepare self-assembling polythiophenes. Researchers found that polar side-chains, such as ether groups, ester groups, and carboxylic- or hydroxyl-terminated alkyl groups, could be incorporated into

(C) 2002 IUPAC, Pure and Applied Chemistry 74, 2031-2044 
the polythiophenes as a side-chain to give hydrophilic character and that the resulting polythiophenes formed a stable monolayer at the air-water interface by themselves [25-30]. It is believed that the introduced hydrophilic character allows the polythiophenes to interact favorably with the water surface that was absent in the case of poly(alkylthiophenes) [20]. Analysis of their pressure-area isotherm implied that the thiophene rings of the polymer backbone were free from strong $\pi$-aggregation by lying cofacial to the air-water interface with a tilt angle. However, in contrast to this hydrophilic effect, it was reported that fluorinated alkyl side-chains that are more hydrophobic and rigid than alkyl chains also produced a stable monolayer of a poly(alkylthiophene) without mixing with fatty acids [31].

Thanks to the development of chemistry, to obtain regioregular alternating amphiphilic polythiophenes, much better control of molecular orientation of polythiophenes at the air-water interface is now available [32-36]. The amphiphilic nature generated from alternating hydrophobic and hydrophilic side-chains along the polymer backbone induced the polythiophenes to orient their $\pi$-plane perpendicular to the air-water interface. In addition, the regioregular structure with $>95 \%$ of head-to-tail coupling enhanced the packing of side-chains. These effects produced highly oriented well-packed Langmuir films of polythiophenes. Grazing-incidence X-ray diffraction and X-ray reflectivity measurements on the monolayer of the amphiphilic regioregular polythiophenes showed that their Langmuir films are 2-dimensional polycrystalline monolayers with card-packing geometry. These monolayers were selectively transferred onto a gold-coated silicon wafer with prepatterned hydrophobic and hydrophilic areas to demonstrate the ability to fabricate polythiophene microchips. Highly oriented $\pi$-stacked thiophene layers have very important implications for realizing a high-performance FET made of polythiophenes because the molecular ordering is critical to the charge transport properties as recently demonstrated [37]. The charge transport mobility was measured to be orders of magnitude faster in the direction normal to the $\pi$-plane as compared to the direction parallel to the $\pi$-plane.

Whereas only one edge of the thiophene ring of polythiophenes can be modified with amphiphilic side-chains, the phenyl ring of PPEs has two available sides for amphiphilic decoration, providing more room for delicate amphiphilic design. For example, both sides can be modified with either hydrophobic side-chains or hydrophilic side-chains, or alternatively one side with hydrophobic and the other side with hydrophilic moieties. Four amphiphilic phenyleneethynylene building blocks were designed to have preferred orientations at the air-water interface, and their initial equilibrium orientations could be converted to different orientations by applying surface pressure. Unique combinations of these building blocks yielded polymers in three interconvertible structures with different conformations and/or interpolymer orientations at the air-water interface (Fig. 1, p. 2035) [38]. Applying mechanical force to the monolayers precisely controlled the conformation of each individual polymer chain and/or interpolymer interactions.

The LB method also produced nanowires of conjugated polymers, as well as nanostructural films. Polythiophene nanowires were fabricated by compressing a Langmuir monolayer of a regioregular amphiphilic thiophene beyond the maximum surface pressure that the monolayer can sustain. The folded Langmuir monolayer produced highly aligned nanowires [33]. Well-aligned nanowires were also observed in the transferred monolayer of a PPE. A Langmuir monolayer of a PPE was transferred to a hydrophobic substrate at a low surface pressure. The resulting monolayer LB film had a high-energy hydrophilic surface, and weakly anchored onto a hydrophobic substrate. The driving force to relieve the high surface energy consequently reorganized the monolayer into nanowires. Because the PPE was aligned along the dipping direction during the transfer, due to the flow field and cycles of mechanical annealing on the monolayer prior to the transfer, the nanowires on the substrate were also highly aligned along the dipping direction. Moreover, changing the dipping direction $90^{\circ}$ for the second layer produced bilayer grid films of the nanowires to demonstrate the ability to control the nanostructure of PPEs (Fig. 2, p. 2035) [39].

Other conjugated polymers including PPV, polypyrrole, and polyanilines also have been reported to be practically suitable for the LB application [40-51]. 


\section{Block copolymers}

Block copolymers are fascinating materials. The order-disorder transition due to the phase separation phenomena between chemically connected immiscible blocks self-assembles block copolymers into ordered structural films [52-55]. To realize ordered films, each block has to be larger than a certain minimum molecular weight and has a narrow molecular weight distribution. Living polymerization methods and ring-opening metathesis have been widely used to produce block copolymers to satisfy the requirements. The microphase-separated morphology can be controlled to be a sphere, cylinder, lamellae, or bicontinuous phase depending on the length, macroscopic shape, and the ratio of each block. Therefore, a block copolymer is a promising approach to produce ordered structural conjugated polymer films. However, it is a great challenge to synthesize a conjugated block copolymer because conventional methods used for block copolymer preparation generally cannot be applied for conjugated polymer synthesis. Hence, there are, as of yet, few reports concerning conjugated block copolymers.

The majority of the reported conjugated block copolymers are rod-coil-type polymers. Synthesis of these conjugated block copolymers generally started with the preparation of end-modified conjugated rod blocks that subsequently reacted with a separately prepared end-functionalized nonconjugated coil block. Alternatively, the end-modified conjugated rod blocks could initiate a living polymerization of nonconjugated coil blocks. Hadziioannou and coworkers synthesized an aldehyde end-functionalized PPV that subsequently reacted with a Grignard reagent containing TEMPO (tetramethylpiperidine- $N$ oxide) group to form a macro-initiator (the PPV-TEMPO). As the flexible second block, either homo polystyrene or a random copolymer of polystyrene/p-chloromethylstyrene was grown from the TEMPO functionality by living radical polymerization. The chlorostyrene functionality was then further reacted with $\mathrm{C}_{60}$, a well-known electron acceptor, to make the final rod-coil conjugated block copolymer for photovoltaic cell applications [56-60]. The spin-cast films of the resulting rod-coil block copolymers produced a microphase-separated morphology without long-range order, whereas drop-cast films of the same copolymer from $\mathrm{CS}_{2}$ solution generated well-ordered microporous films. Similar microporous honeycomb morphology was observed in a drop-cast film of a poly(p-phenylene)-b-polystyrene $[61,62]$. These microhoneycomb holes have no precedent among conventional coil-coil block copolymers, suggesting that the morphology may not be directed from the microphase separation of the block copolymers, as the authors suggested, but is likely derived from condensed water droplets on the dropcast films during the evaporation of $\mathrm{CS}_{2}[63]$.

On the other hand, Jenekhe and coworkers reported microphase-separation-driven ordered microporous films of poly(phenylquinoline)- $b$-polystyrene, a conjugated rod-coil block copolymer [64]. This microporous structure originated from the micelle formation of the block copolymer in $\mathrm{CS}_{2}$. Because $\mathrm{CS}_{2}$ is a good solvent for polystyrene, the polystyrene blocks formed coronae and the poly(phenylquinoline) rod blocks formed hollow cores. As the solvent evaporated, the micelles coagulated into a highly iridescent ordered microporous film (Fig. 3). When the same copolymer was dissolved in trifluoroacetic acid, the good solvent for the rod block, the poly(phenylquinoline) rod blocks formed coronae and the polystyrene blocks formed cores [65]. Varying the cosolvent composition of trifluoroacetic acid/dichloromethane directed the formation of four different micelle morphologies such as sphere, lamellae, cylinder, and vesicle.

A thiophene moiety also has been incorporated into rod-coil block copolymers. However, when the length of thiophene block was short, no microphase morphology was observed from the resulting oligothiophene- $b$-polystyrene [66,67]. Recently, diblock and triblock copolymers containing highmolecular-weight poly(alkylthiophene) were successfully prepared through atom transfer radical polymerization of styrene at the end-functional group of the poly(alkylthiophene) block [68]. The thin-layer drop-cast films of the resulting poly(3-hexylthiophene)- $b$-polystyrene produced well-defined nanowires with a 30-40-nm lateral spacing that is equivalent to the fully extended rod block length. Nanowire structures also have been found in thin layer films of other conjugated rod-coil block copolymers such

(C) 2002 IUPAC, Pure and Applied Chemistry 74, 2031-2044 


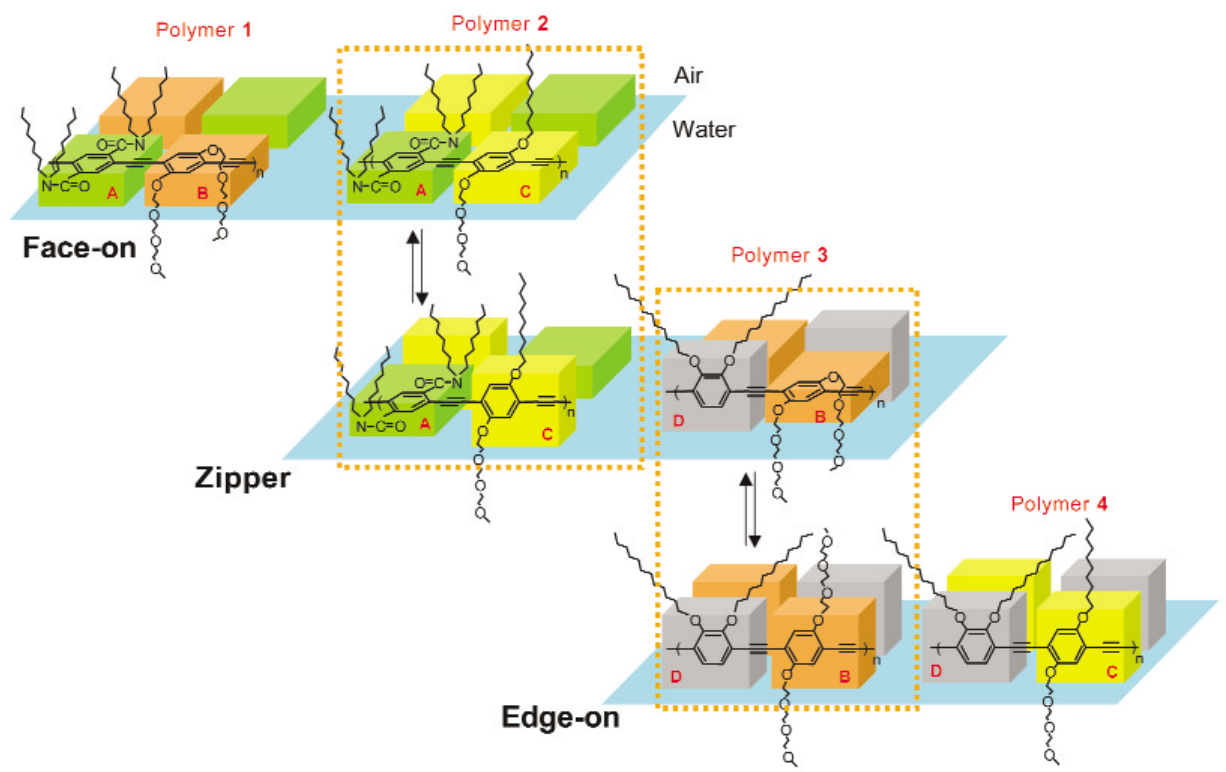

Fig. 1 Conformations and spatial arrangements of the PPEs 1-4 at the air-water interface and their reversible conversion between the three unique structures [38].

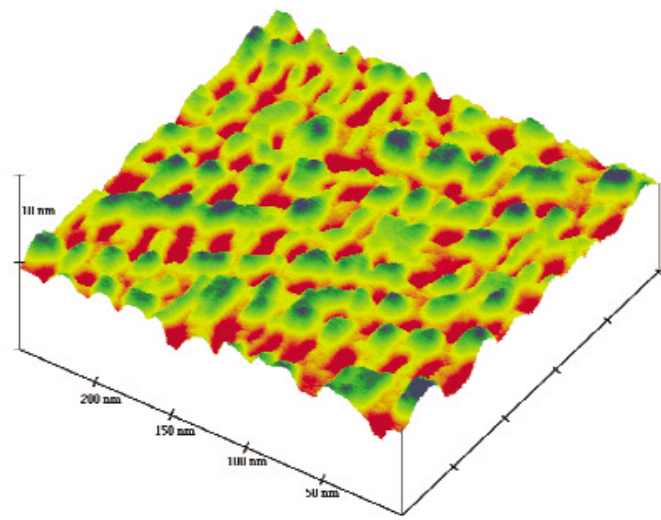

Fig. 2 Atomic force images acquired in tapping mode of 3-D grid pattern nanowires of a PPE [39].

as PPV-b-poly(dimethylsiloxane) [69,70], poly(p-phenylene)- $b$-polystyrene [71], oligo(phenylenevinylene)- $b$-poly(ethyleneoxide) [72], and poly( $p$-phenylene)- $b$-PMMA [71]. This nanowire structure of rod-coil conjugated block copolymers is an unusual morphology in conventional coil-coil block copolymers, possibly derived from the distinct microscopic shape of the blocks and the strong $\pi$-interaction among conjugated rod blocks.

A rod-rod-type conjugated block polymer, polyfluorene- $b$-polyaniline, was also reported. An amino end-functionalized polyfluorene was prepared first. Subsequent polymerization reaction of a polyaniline was initiated at the amine functionality of the polyfluorene block to constitute the second rod block [73]. Thermal treatment on a spin-cast film of the resulting rod-rod block copolymers showed microphase morphology, but without long-range order.

(C) 2002 IUPAC, Pure and Applied Chemistry 74, 2031-2044 


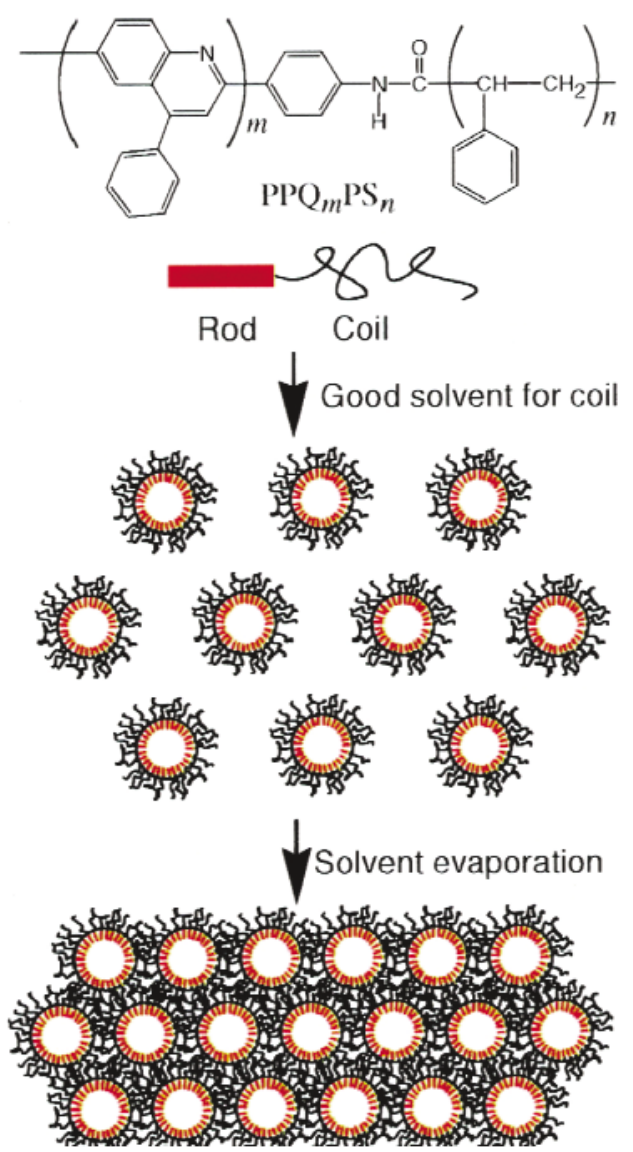

Fig. 3 Schematic illustration of the self-assembly of a rod-coil block copolymer into an ordered microporous structure [64].

Conjugated block copolymers are a promising system to populate a nanoworld of conjugated polymers, provided that there will be improved fundamental understanding of the phase behaviors and development of well-controlled chemistries.

\section{Other organizing methods}

Other organizing methods have been devised for ordering conjugated polymers. It was suggested that the strong $\pi$-interaction force between PPE backbones can be a useful driving force to self-assemble PPEs on solid substrates. A near-saturated solution of a PPE with long alkyl side-chains formed nematic-like textures at the interface of the liquid and the highly oriented pyrolitic graphite (HOPG) [74,75]. Scanning tunneling microscopy (STM) revealed that the polymer backbones and their sidechains lay parallel to an HOPG surface and formed the nematic-like texture. Interestingly, the same polymer self-assembled into nanoribbon architecture either on a mica surface or a glass slide [76,77]. The nanoribbons turned out to be bilayer aggregates of PPEs with their long hydrophobic alkyl sidechains arranged perpendicular to the substrate. The interplay between two competing interaction forces, interpolymer interaction and polymer-substrate interaction, generated the different orderings out of the same polymer. 
By this pseudo-epitaxial-like growth method, a regioregular poly(3-alkylthiophene) was organized on an HOPG surface [78,79]. The STM image on the thin-layer film showed that the polythiophenes were well packed with hairpin-like conformation, despite their polydispersity (Fig. 4). Although polythiophene is considered as a stiff rod-like molecule, modeling studies indicated that seven or eight consecutive thiophenes in an all syn conformation can make a perfect $180^{\circ}$ turn, similar to the $\beta$-turn of proteins. Because the enthalpic penalty for the formation of the less-favorable syn conformation as compared to anti conformation is relatively small, around $3-5 \mathrm{kcal} / \mathrm{mol}$, the regioregular polythiophene constituted the hairpin turn to make a full coverage of the HOPG.

A recently reported approach makes use of conjugated polymer/silica nanocomposite structures. Schwartz et al. infiltrated poly[2-methoxy,5-(2'-ethyl-hexyloxy)-p-phenylene-vinylene] (MEH-PPV) into preformed, oriented, hexagonally arrayed channels of mesoporous silica glass. Because of the channel size, each channel can accommodate only one MEH-PPV, producing well-aligned, isolated arrays of conjugated polymers. The energy migration along the isolated polymer chains in the channels and randomly oriented chain ends outside the channels, was studied with polarized femtosecond spectroscopy to reveal that interpolymer energy transport is much faster than intrapolymer energy transport in the case of MEH-PPV [80,81]. To avoid the difficulty in infiltration of large-molecular-weight polymers into small channels, and to realize various structural nanocomposites, Brinker and coworkers devised a self-assembly system in which the nanoporous silica framework was constituted simultaneously with the polymerization of a conjugated polymer [82]. Silicic acids and surfactant molecules composed of diacetylene moiety were directed to form mesoscopically ordered structures, such as cubicpacked spheres, hexagonally arranged cylinders, and lamellae, depending on the head size of the surfactant molecules. Subsequent topochemical polymerization of diacetylenes and parallel acid-catalyzed condensation reaction of siloxane produced various mesoscopically ordered polydiacetylene/silica nanocomposites.

(a)

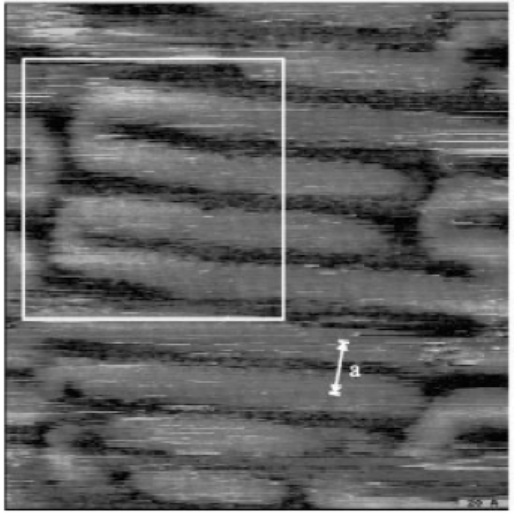

(b)

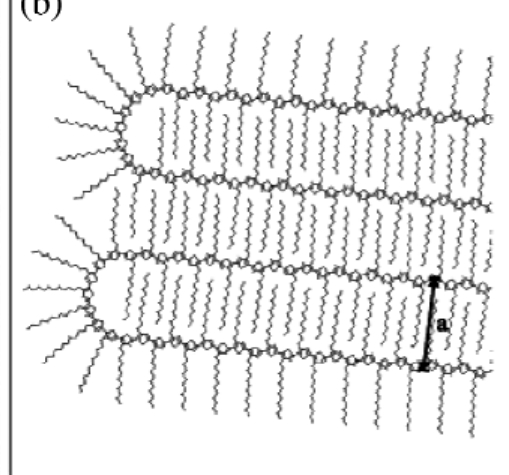

Fig. 4 (a) STM image of a poly(3-dodecylthiophene) on HOPG $(20 \times 20 \mathrm{~nm})$. (b) Simulated model of the poly(3-dodecylthiophene) corresponding to the white box in the STM image [78].

\section{INTRAMOLECULAR EFFECTS IN THE PHOTOPHYSICAL PROPERTIES OF CONJUGATED POLYMERS}

The attractive optoelectronic properties of conjugated polymers stems from their extended $\pi$-bond system along the backbone. The effective conjugation length of a conjugated polymer is strongly affected by the conformation of the conjugated polymer because the degree of the p-orbital overlap in the extended $\pi$-bond is determined by the torsion angle of the backbone. Therefore, the optoelectronic 
properties of conjugated polymers such as optical band-gap and ionization potential are decisively influenced by the conformation of the polymers.

There have been theoretical and experimental studies on conformational effects in optoelectronic properties of conjugated polymers including PPVs, polythiophenes, poly( $p$-phenylenes), and PPEs [83-91]. Many studies have been done in various solvent systems at different temperatures to manipulate the interaction between the solvent and the side-chains of conjugated polymers in solution. This varying interaction produces different steric hindrance among side-chains, inducing conformational changes of a polymer. However, the resulting conformations are not predictable, and the effects of solvent and temperature on photophysical properties cannot be excluded. Therefore, if we devise a method to twist an isolated polymer backbone by applying a pressure, it would specifically elucidate conformational effects. Recently, a series of PPEs constituted with unique combinations of surfactant sidechains provided a clear picture of the conformational effects by adapting their conformation in response to an imposed surface pressure at the air-water interface (Fig. 1) [38,92]. The torsion angle was precisely controlled by varying the surface pressure exerted on the Langmuir films. A unique in situ UV-vis and fluorescence spectroscopy set-up allowed the researchers to monitor the conformational effects on photophysical properties at various controlled conformations.

All of the results obtained from numerous studies have been in good agreement with each other, indicating that the optical band-gap has the minimum value in the planar conformation of a conjugated polymer backbone by maximizing p-orbital overlap along the backbone. Therefore, the absorption and emission $\lambda_{\max }$ could be tuned by controlling the conformation of a conjugated polymer. For example, bridging consecutive units of a conjugated polymer with covalent bonds fixed the torsion angle between the rings, producing a ladder-like polymer whose absorption $\lambda_{\max }$ shifted to a longer wavelength $[90,91,93,94]$. This band-gap engineering is important in practical applications of conjugated polymers, especially for LED applications $[89,95]$. The tunable properties also have been utilized in developing colorimetric sensors. Environmental stimuli such as heat (thermochromism), solvents (solvatochromism), external pressure (piezochromism), light (photochromism), or the presence of ions (ionochromism), can trigger conformational changes in conjugated polymers such as PPVs, polythiophenes, polysilanes, and polydiacetylenes, producing noticeable color changes as a sensory signal $[96,97]$.

A conjugated polymer chain can be considered as a series of connected chromophores with various effective conjugation lengths [98]. Therefore, an exciton travels along the energy gradient established among the chain segments with statistically distributed effective conjugation lengths. In an extended planar conformation, an exciton migrates randomly along the rather homogeneous energy surface, producing fluorescent emission from multiple sites. On the other hand, if there is a distinct lower energy site on the backbone, an exciton will effectively funnel to the site, emitting from the lower energy site [99-101]. Therefore, conformation of a single polymer also affects energy transport properties, exciton lifetime, and the quantum yield of single chains, as well as the color change. Increased quantum efficiency was observed as the PPV chain became stiffer, and ladder-type oligo- $p$-phenylenes showed reduced exciton lifetime as compared to nonplanar oligo- $p$-phenylenes counterparts $[102,103]$.

\section{INTERMOLECULAR EFFECTS IN THE PHOTOPHYSICAL PROPERTIES OF CONJUGATED POLYMERS}

Intermolecular interactions have a large impact on the optoelectronic properties of conjugated polymers as numerous theoretical [104-109] and experimental [38,92,110-130] research papers have revealed. Even though there have been some exceptions $[110,115]$, it is generally believed that strong intermolecular interactions between conjugated polymers form weakly emissive interchain species, for example, a ground-state aggregate and an excimer, and lead to spectral red-shift and reduced quantum yield $[114,118]$. 
By definition, an excimer is a dimer of two molecules, one in its ground state and the other in its excited state. Because an excimer does not have ground-state interactions to form a complex molecule it is not directly excitable. On the other hand, a ground-state aggregate is a dimer of cofacially aggregated conjugated molecules with ground-state chain interactions. Therefore, a ground-state aggregate forms a new chromophore that is directly excitable. The understanding of the ground-state aggregation, in the case of small molecules, has been well established due to the manageable intermolecular orientation and packing, in addition to straightforward theoretical modeling. When two chromophores form a card pack-like orientation (H-aggregate), the resulting excitonic splitting produces a forbidden lower-energy band and an allowed higher-energy band, consequently inducing blue-shift. In contrast, a J-aggregate (end-to-end orientation of chromophores) produces a red-shift due to the resulting allowed lower-energy band and a forbidden higher-energy band [104,131]. However, in the case of conjugated polymers the ambiguity in defining chromophores, the amorphous character, and the large molecular weight have prevented systematic investigation.

Even though the detailed identity of interchain species is controversial, a generally accepted understanding is that interchain species produce lower-energy sites where singlet excitons efficiently migrate to emit red-shifted light. This picture was supported by the numerous experimental results that photoluminescence (PL) decay of an isolated conjugated polymer in dilute solution is nearly single exponential, whereas that of solid films is nonexponential with a long-lived component. The long-lived component has been mostly attributed to either an excimer or a ground-state aggregate formed by strong intermolecular interactions. However, because an excimer and a ground-state aggregate have common features such as red-shifted emission, low quantum yield, and long-lived PL decay, usually it is not clear whether intermolecular interactions create an excimer or a ground-state aggregate. Consequently, several papers have used "intermolecular species" instead of distinguishing the contribution of the two different dimers. In addition, "aggregates" have commonly been used in the literature to describe both the ground-state aggregates and morphological aggregates, possibly causing misunderstanding.

Ground-state aggregation requires the more stringent condition that two conjugate polymers have to be packed cofacially and closely enough to establish ground-state interactions. Even if conjugated polymers in spatial proximity do not meet the ground-state aggregation condition, they still could form an excimer because they are known to have a planar structure in the excited state, promoting intermolecular interactions. Therefore, many papers have attributed the more feasible excimer formation to the red-shifted PL spectra with low quantum yield observed in conjugated polymer films $[113,114,119,121-123]$. On the other hand, several research papers concluded that red-shifted featureless PL spectra with low quantum yield are originated from ground-state aggregates, not from excimers. Blatchford and coworkers studied morphology-dependent PL properties of several pyridine-based conjugated polymers $[116,117]$. Time-resolved luminescence studies and near-field scanning optical microscopy (NSOM) analysis convinced the authors of the fact that the fast diffusion of intrachain excitons to the directly excitable ground-state aggregates in a localized partially aligned region produces the highly nonexponential PL decay composed of a rapid initial decay followed by a long-lived component. Directly excitable distinct absorption bands also have been observed in films of other conjugated polymers such as poly(fluorenes) [124,125], ladder-type poly(p-phenylenes) [126,127], MEH-PPV $[128,129]$, and PPEs $[38,92,110-112]$, indicating that ground-state aggregates are commonly present in conjugated polymer films and contribute to the low quantum yield.

The evident difference between a ground-state aggregate and an excimer is the presence of a directly excitable red-shifted absorption band. This fact has been used to discriminate the presence of a ground-state aggregation in conjugated polymer films. However, recently, Bunz and coworkers suggested that the additional absorption band observed in the PPE films that is absent in solution is not due to the ground-state aggregation but to the planarization of PPE backbones induced by morphological aggregations $[83,84,132]$. Their claim is based on the fact that planarization is a necessary condition for the formation of a ground-state aggregate. As discussed in a previous section, planarization of a conjugated polymer backbone maximizes the effective conjugation length by enhancing p-orbital overlap.

(C) 2002 IUPAC, Pure and Applied Chemistry 74, 2031-2044 
Experimentally, it was clearly shown that planarization of PPEs indeed produced a red-shifted absorption band [38,92]. However, it was also demonstrated that co-present ground-state aggregation can induce the red-shift even further. Moreover, the presence of the red-shifted absorption band could be manipulated by introducing macrocycles on the polymer backbone or by changing the side-chain bulk of PPEs that determines the interpolymer spacing in the ordered structures [38,92,111,112]. In addition, detailed photophysical studies of the solid solutions of PPE/PMMA showed the appearance and increase of a red-shifted absorption band as the average interpolymer distance is reduced. When a solid solution had both a monomer-like band and the red-shifted absorption band, excitation at different wavelengths resulted in intensity redistribution between a monomer-like emission band and a redshifted emission band. These results strongly indicated that intermolecular ground-state aggregation is also responsible for the red-shifted additional absorption band [92,110]. Moreover, the role of groundstate aggregation in producing a red-shifted absorption band was evidently supported by photophysical studies of 1,4-diethynyl-2-fluorobenzene. This small molecule analogous of PPE is the simplest arylethynyl compound free from the planarization effect [130]. Diffuse reflectance and fluorescence measurements on crystals of the compound clearly showed an additional large red-shifted absorption and emission bands resulting from intermolecular interactions.

In any case, strong intermolecular interactions deteriorate the emission properties of conjugated polymers. To prevent the self-quenching due to the strong intermolecular interactions, several effective methods have been devised. Bulky side-groups were introduced and successfully separated conjugated polymer backbones to enhance emission yield in the solid state [133,134]. A dendrimer approach is also promising because the highly branched structure likely prevents close cofacial intermolecular aggregation [135]. Introducing cis-linkage in a PPV also realized high emission yield by causing the PPV backbone to deviate from a rod-like structure, consequently disturbing chain packing [136]. Another interesting approach is the spiro-type design of conjugated molecules [137-139]. Covalently bonded orthogonally oriented conjugated molecules (spiro-type) were reported to have high luminescence yield in the solid state. Even though the result was obtained from low-molecular-weight conjugated molecules, the design principle is applicable to polymeric analogs.

In this review paper, recent developments in assembly methods to fabricate ordered conjugated polymer films, and intra- and intermolecular effects on the photophysical properties of conjugated polymers are discussed. The intramolecular conformation of a single conjugated polymer is decisive in determining its absorption and emission $\lambda_{\max }$ by affecting the effective conjugation length through altering the degree of p-orbital overlap along the conjugated polymer backbone. Strong intermolecular interactions are detrimental to the emission properties of conjugated polymers by producing intermolecular species with low quantum yield. The intramolecular and intermolecular effects also strongly affect other properties such as energy and charge transport properties as well as photophysical properties of conjugated polymers. As conjugated polymers have become increasingly important active components in many optoelectronic devices, effective control of intra- and intermolecular effects through structural control at the molecular level and through patterning [140] are necessary to realize optimum device performance.

\section{REFERENCES}

1. G. Decher. Science 277, 1232-1237 (1997).

2. M. Ferreira, J. H. Cheung, M. F. Rubner. Thin Solid Films 244, 806-809 (1994).

3. M. Ferreira and M. F. Rubner. Macromolecules 28, 7107-7114 (1995).

4. J. H. Cheung, A. F. Fou, M. F. Rubner. Thin Solid Films 244, 985-989 (1994).

5. A. C. Fou and M. F. Rubner. Macromolecules 28, 7115-7120 (1995).

6. J. H. Cheung, W. B. Stockton, M. F. Rubner. Macromolecules 30, 2712-2716 (1997).

7. W. B. Stockton and M. F. Rubner. Macromolecules 30, 2717-2725 (1997). 
8. H. P. Hong, D. Davidov, Y. Avny, H. Chayet, E. Z. Faraggi, R. Neumann. Adv. Mater. 7, 846-849 (1995).

9. H. Mattoussi, L. H. Radzilowski, B. O. Dabbousi, E. L. Thomas, M. G. Bawendi, M. F. Rubner. J. Appl. Phys. 83, 7965-7974 (1998).

10. H. Mattoussi, M. F. Rubner, F. Zhou, J. Kumar, S. K. Tripathy, L. Y. Chiang. Appl. Phys. Lett. 77, 1540-1542 (2000).

11. J. D. Hong, D. Kim, K. Cha, J. I. Jin. Synth. Met. 84, 815-816 (1997).

12. M. F. Durstock, B. Taylor, R. J. Spry, L. Chiang, S. Reulbach, K. Heitfeld, J. W. Baur. Synth. Met. 116, 373-377 (2001).

13. H. L. Wang, D. W. McBranch, R. J. Donohoe, S. Xu, B. Kraabel, L. H. Chen, D. Whitten, R. Helgeson, F. Wudl. Synth. Met. 121, 1367-1368 (2001).

14. H. L. Wang, D. W. McBranch, V. I. Klimov, R. Helgeson, F. Wudl. Chem. Phys. Lett. 315, 173-180 (1999).

15. D. T. McQuade, A. H. Hegedus, T. M. Swager. J. Am. Chem. Soc. 122, 12389-12390 (2000).

16. G. Wegner. Thin Solid Films 216, 105-116 (1992).

17. I. Watanabe, K. Hong, M. F. Rubner. J. Chem. Soc., Chem. Commun. 123-124 (1989).

18. I. Watanabe, K. Hong, M. F. Rubner, I. H. Loh. Synth. Met. 28, C473-C480 (1989).

19. A. Chyla, S. Kucharski, R. Janik, J. Sworakowski, M. Bienkowski. Thin Solid Films 285, 496-499 (1996).

20. I. Watanabe, K. Hong, M. F. Rubner. Langmuir 6, 1164-1172 (1990).

21. M. Rikukawa, M. Nakagawa, H. Abe, K. Ishida, K. Sanui, N. Ogata. Thin Solid Films 273, 240-244 (1996).

22. M. Rikukawa, M. Nakagawa, K. Ishida, H. Abe, K. Sanui, N. Ogata. Thin Solid Films 285, 636-639 (1996).

23. M. Rikukawa, M. Nakagawa, Y. Tabuchi, K. Sanui, N. Ogata. Synth. Met. 84, 233-234 (1997).

24. M. Rikukawa, Y. Tabuchi, K. Ochiai, K. Sanui, N. Ogata. Thin Solid Films 329, 469-472 (1998).

25. L. Belobrzeckaja, G. Bajo, A. Bolognesi, M. Catellani. Synth. Met. 84, 195-196 (1997).

26. A. Bolognesi, G. Bajo, D. Comoretto, P. Elmino, S. Luzzati. Thin Solid Films 299, 169-172 (1997).

27. A. Bolognesi, G. Bajo, Z. Geng, W. Porzio, F. Speroni. Thin Solid Films 243, 683-686 (1994).

28. A. Bolognesi, F. Bertini, G. Bajo, A. Provasoli, D. Villa, O. Ahumada. Thin Solid Films 289, 129-132 (1996).

29. S. Sagisaka, M. Ando, T. Iyoda, T. Shimidzu. Thin Solid Films 230, 65-69 (1993).

30. C. L. Callender, C. A. Carere, G. Daoust, M. Leclerc. Thin Solid Films 204, 451-457 (1991).

31. L. Robitaille and M. Leclerc. Macromolecules 27, 1847-1851 (1994).

32. T. Bjornholm, D. R. Greve, N. Reitzel, T. Hassenkam, K. Kjaer, P. B. Howes, N. B. Larsen, J. Bogelund, M. Jayaraman, P. C. Ewbank, R. D. McCullough. J. Am. Chem. Soc. 120, 7643-7644 (1998).

33. T. Bjornholm, T. Hassenkam, D. R. Greve, R. D. McCullough, M. Jayaraman, S. M. Savoy, C. E. Jones, J. T. McDevitt. Adv. Mater. 11, 1218-1221 (1999).

34. D. R. Greve, N. Reitzel, T. Hassenkam, J. Bogelund, K. Kjaer, P. B. Howes, N. B. Larsen, M. Jayaraman, R. D. McCullough, T. Bjornholm. Synth. Met. 102, 1502-1505 (1999).

35. N. Reitzel, D. R. Greve, K. Kjaer, P. B. Hows, M. Jayaraman, S. Savoy, R. D. McCullough, J. T. McDevitt, T. Bjornholm. J. Am. Chem. Soc. 122, 5788-5800 (2000).

36. T. Bjornholm, T. Hassenkam, N. Reitzel. J. Mater. Chem. 9, 1975-1990 (1999).

37. H. Sirringhaus, P. J. Brown, R. H. Friend, M. M. Nielsen, K. Bechgaard, B. M. W. LangeveldVoss, A. J. H. Spiering, R. A. J. Janssen, E. W. Meijer, P. Herwig, D. M. de Leeuw. Nature 401, 685-688 (1999).

38. J. Kim and T. M. Swager. Nature 411, 1030-1034 (2001). 
39. J. Kim, S. K. McHugh, T. M. Swager. Macromolecules 32, 1500-1507 (1999).

40. A. T. Royappa and M. F. Rubner. Langmuir 8, 3168-3177 (1992).

41. T. W. Kim, H. S. Lee, J. H. Lee, J. W. Park, D. Y. Kang. Synth. Met. 121, 1427-1428 (2001).

42. Y. K. Kim, K. S. Kim, W. H. Kang, S. S. Yang, B. C. Sohn. Thin Solid Films 312, 291-294 (1998).

43. A. P. Wu, T. Fujiwara, M. Kakimoto, Y. Imai, T. Kubota, M. Iwamoto. React. Func. Polym. 30, 361-365 (1996).

44. A. P. Wu and M. A. Kakimoto. Adv. Mater. 7, 812-814 (1995).

45. A. P. Wu, S. Yokoyama, S. Watanabe, M. Kakimoto, Y. Imai, T. Araki, K. Iriyama. Thin Solid Films 244, 750-753 (1994).

46. Z. K. Wu, S. X. Wu, Y. Q. Liang. Langmuir 17, 7267-7273 (2001).

47. Z. K. Wu, S. X. Wu, Z. H. Lu, Y. Q. Liang. J. Colloid Interface Sci. 251, 125-130 (2002).

48. A. Marletta, F. A. Castro, D. Goncalves, O. N. Oliveira, R. M. Faria, F. E. G. Guimaraes. Synth. Met. 121, 1447-1448 (2001).

49. A. Marletta, D. Goncalves, O. N. Oliveira, R. M. Faria, F. E. G. Guimaraes. Macromolecules 33, 5886-5890 (2000).

50. J. H. Cheung and M. F. Rubner. Thin Solid Films 244, 990-994 (1994).

51. M. Rikukawa and M. F. Rubner. J. Macromol. Sci., Pure Appl. Chem. A31, 793-803 (1994).

52. E. L. Thomas and R. L. Lescanec. Philos. Trans. R. Soc. London Ser. A 348, 149-166 (1994).

53. S. Forster and M. Antonietti. Adv. Mater. 10, 195-217 (1998).

54. F. S. Bates and G. H. Fredrickson. Phys. Today 52, 32-38 (1999).

55. F. S. Bates and G. H. Fredrickson. Annu. Rev. Phys. Chem. 41, 525-557 (1990).

56. U. Stalmach, B. de Boer, C. Videlot, P. F. van Hutten, G. Hadziioannou. J. Am. Chem. Soc. 122, 5464-5472 (2000).

57. B. de Boer, U. Stalmach, C. Melzer, G. Hadziioannou. Synth. Met. 121, 1541-1542 (2001).

58. B. de Boer, U. Stalmach, H. Nijland, G. Hadziioannou. Adv. Mater. 12, 1581-1583 (2000).

59. B. de Boer, U. Stalmach, P. F. van Hutten, C. Melzer, V. V. Krasnikov, G. Hadziioannou. Polymer 42, 9097-9109 (2001).

60. G. Hadziioannou. MRS Bull. 27, 456-460 (2002).

61. G. Widawski, M. Rawiso, B. Francois. Nature 369, 387-389 (1994).

62. X. F. Zhong and B. Francois. Makromol. Chemie, Rapid Commun. 9, 411-416 (1988).

63. M. Srinivasarao, D. Collings, A. Philips, S. Patel. Science 292, 79-83 (2001).

64. S. A. Jenekhe and X. L. Chen. Science 283, 372-375 (1999).

65. S. A. Jenekhe and X. L. Chen. Science 279, 1903-1907 (1998).

66. R. S. Saunders, R. E. Cohen, R. R. Schrock. Macromolecules 24, 5599-5605 (1991).

67. W. J. Li, T. Maddux, L. P. Yu. Macromolecules 29, 7329-7334 (1996).

68. J. S. Liu, E. Sheina, T. Kowalewski, R. D. McCullough. Angew. Chem., Int. Ed. 41, 329-332 (2002).

69. P. Leclere, A. Calderone, D. Marsitzky, V. Francke, Y. Geerts, K. Mullen, J. L. Bredas, R. Lazzaroni. Adv. Mater. 12, 1042-1046 (2000).

70. P. Leclere, A. Calderone, D. Marsitzky, V. Francke, K. Mullen, J. L. Bredas, R. Lazzaroni. Synth. Met. 121, 1295-1296 (2001).

71. P. Leclere, V. Parente, J. L. Bredas, B. Francois, R. Lazzaroni. Chem. Mater. 10, 4010-4014 (1998).

72. H. B. Wang, H. H. Wang, V. S. Urban, K. C. Littrell, P. Thiyagarajan, L. P. Yu. J. Am. Chem. Soc. 122, 6855-6861 (2000).

73. C. Schmitt, H. G. Nothofer, A. Falcou, U. Scherf. Macromol. Rapid Commun. 22, 624-628 (2001). For relevant examples of rod-rod block copolymers, see: X. L. Chen and S. A. Jenekhe. Macromolecules 29, 6189-6192 (1969) and X. L. Chen and S. A. Jenekhe. Appl. Phys. Lett. 70, 487-489 (1997).

74. P. Samori, V. Francke, K. Mullen, J. P. Rabe. Chem.—Eur. J. 5, 2312-2317 (1999).

(C) 2002 IUPAC, Pure and Applied Chemistry 74, 2031-2044 
75. P. Samori, N. Severin, K. Mullen, J. P. Rabe. Adv. Mater. 12, 579-582 (2000).

76. P. Samori, V. Francke, K. Mullen, J. P. Rabe. Thin Solid Films 336, 13-15 (1998).

77. P. Samori, I. Sikharulidze, V. Francke, K. Mullen, J. P. Rabe. Nanotechnology 10, 77-80 (1999).

78. E. Mena-Osteritz. Adv. Mater. 14, 609-616 (2002).

79. E. Mena-Osteritz, A. Meyer, B. M. W. Langeveld-Voss, R. A. J. Janssen, E. W. Meijer, P. Bauerle. Angew. Chem., Int. Ed. 39, 2680-2684 (2000).

80. T. Q. Nguyen, J. J. Wu, V. Doan, B. J. Schwartz, S. H. Tolbert. Science 288, 652-656 (2000).

81. B. J. Schwartz, T. Q. Nguyen, J. J. Wu, S. H. Tolbert. Synth. Met. 116, 35-40 (2001).

82. Y. F. Lu, Y. Yang, A. Sellinger, M. C. Lu, J. M. Huang, H. Y. Fan, R. Haddad, G. Lopez, A. R. Burns, D. Y. Sasaki, J. Shelnutt, C. J. Brinker. Nature 410, 913-917 (2001).

83. M. Levitus, K. Schmieder, H. Ricks, K. D. Shimizu, U. H. F. Bunz, M. A. Garcia-Garibay. J. Am. Chem. Soc. 123, 4259-4265 (2001).

84. T. Miteva, L. Palmer, L. Kloppenburg, D. Neher, U. H. F. Bunz. Macromolecules 33, 652-654 (2000).

85. S. Rughooputh, S. Hotta, A. J. Heeger, F. Wudl. J. Polym. Sci., Part B: Polym. Phys. 25, 1071-1078 (1987).

86. P. Barta, F. Cacialli, R. H. Friend, W. R. Salaneck, M. Zagorska, A. Pron. Synth. Met. 101, 296-297 (1999).

87. J. L. Bredas, G. B. Street, B. Themans, J. M. Andre. J. Chem. Phys. 83, 1323-1329 (1985).

88. B. Themans, W. R. Salaneck, J. L. Bredas. Synth. Met. 28, C359-C364 (1989).

89. B. L. Lucht, S. S. H. Mao, T. D. Tilley. J. Am. Chem. Soc. 120, 4354-4365 (1998).

90. Y. X. Yao, Q. T. Zhang, J. M. Tour. Macromolecules 31, 8600-8606 (1998).

91. Q. T. Zhang and J. M. Tour. J. Am. Chem. Soc. 119, 9624-9631 (1997).

92. J. Kim, I. A. Levitsky, D. T. McQuade, T. M. Swager. J. Am. Chem. Soc. 124, 7710-7718 (2002).

93. U. Scherf and E. J. W. List. Adv. Mater. 14, 477-487 (2002).

94. U. Scherf and K. Mullen. "The Synthesis of Ladder Polymers", in Synthesis and Photosynthesis, Vol. 123, pp. 1-40, Springer, Berlin (1995).

95. J. Roncali. Chem. Rev. 97, 173-205 (1997).

96. B. Wang and M. R. Wasielewski. J. Am. Chem. Soc. 119, 12-21 (1997).

97. M. Leclerc. Adv. Mater. 11, 1491-1498 (1999).

98. H. Bassler, M. Gailberger, R. F. Mahrt, J. M. Oberski, G. Weiser. Synth. Met. 49, 341-352 (1992).

99. T. Huser, M. Yan, L. J. Rothberg. Proc. Natl. Acad. Sci. U.S.A. 97, 11187-11191 (2000).

100. S. Heun, R. F. Mahrt, A. Greiner, U. Lemmer, H. Bassler, D. A. Halliday, D. D. C. Bradley, P. L. Burn, A. B. Holmes. J. Phys.: Condens. Matter 5, 247-260 (1993).

101. T. Huser and M. Yan. J. Photochem. Photobiol., A 144, 43-51 (2001).

102. U. Rant, U. Scherf, M. Rehahn, P. Galda, J. L. Bredas, E. Zojer. Synth. Met. 127, 241-245 (2002).

103. C. L. Gettinger, A. J. Heeger, J. M. Drake, D. J. Pine. J. Chem. Phys. 101, 1673-1678 (1994).

104. J. Cornil, D. Beljonne, J. P. Calbert, J. L. Bredas. Adv. Mater. 13, 1053-1067 (2001).

105. J. L. Bredas, J. Cornil, D. Beljonne, D. dos Santos, Z. G. Shuai. Acc. Chem. Res. 32, 267-276 (1999).

106. J. Cornil, D. A. dos Santos, X. Crispin, R. Silbey, J. L. Bredas. J. Am. Chem. Soc. 120, 1289-1299 (1998).

107. J. Cornil, A. J. Heeger, J. L. Bredas. Chem. Phys. Lett. 272, $463-470$ (1997).

108. R. Jakubiak, C. J. Collison, W. C. Wan, L. J. Rothberg, B. R. Hsieh. J. Phys. Chem. A 103, 2394-2398 (1999).

109. S. Tretiak, A. Saxena, R. L. Martin, A. R. Bishop. J. Phys. Chem. B 104, 7029-7037 (2000).

110. R. Deans, J. Kim, M. R. Machacek, T. M. Swager. J. Am. Chem. Soc. 122, 8565-8566 (2000).

111. J. Kim, D. T. McQuade, S. K. McHugh, T. M. Swager. Angew. Chem., Int. Ed. 39, 3868-3872 (2000).

112. D. T. McQuade, J. Kim, T. M. Swager. J. Am. Chem. Soc. 122, 5885-5886 (2000).

(C) 2002 IUPAC, Pure and Applied Chemistry 74, 2031-2044 
113. S. A. Jenekhe. Adv. Mater. 7, 309-311 (1995).

114. S. A. Jenekhe and J. A. Osaheni. Science 265, 765-768 (1994).

115. J. A. Osaheni and S. A. Jenekhe. Macromolecules 27, 739-742 (1994).

116. J. W. Blatchford, T. L. Gustafson, A. J. Epstein, D. A. VandenBout, J. Kerimo, D. A. Higgins, P. F. Barbara, D. K. Fu, T. M. Swager, A. G. MacDiarmid. Phys. Rev. B 54, R3683-R3686 (1996).

117. J. W. Blatchford, S. W. Jessen, L. B. Lin, T. L. Gustafson, D. K. Fu, H. L. Wang, T. M. Swager, A. G. MacDiarmid, A. J. Epstein. Phys. Rev. B 54, 9180-9189 (1996).

118. E. Conwell. Trends Polym. Sci. 5, 218-222 (1997).

119. I. D. W. Samuel, G. Rumbles, C. J. Collison, B. Crystall, S. C. Moratti, A. B. Holmes. Synth. Met. 76, 15-18 (1996).

120. H. Li, D. R. Powell, T. K. Firman, R. West. Macromolecules 31, 1093-1098 (1998).

121. H. Li, D. R. Powell, R. K. Hayashi, R. West. Macromolecules 31, 52-58 (1998).

122. Y. Shi, J. Liu, Y. Yang. J. Appl. Phys. 87, 4254-4263 (2000).

123. C. Weder and M. S. Wrighton. Macromolecules 29, 5157-5165 (1996).

124. J. Teetsov and M. A. Fox. J. Mater. Chem. 9, 2117-2122 (1999).

125. M. Grell, D. D. C. Bradley, X. Long, T. Chamberlain, M. Inbasekaran, E. P. Woo, M. Soliman. Acta Polym. 49, 439-444 (1998).

126. U. Lemmer, S. Heun, R. F. Mahrt, U. Scherf, M. Hopmeier, U. Siegner, E. O. Gobel, K. Mullen, H. Bassler. Chem. Phys. Lett. 240, 373-378 (1995).

127. R. F. Mahrt, T. Pauck, U. Lemmer, U. Siegner, M. Hopmeier, R. Hennig, H. Bassler, E. O. Gobel, P. H. Bolivar, G. Wegmann, H. Kurz, U. Scherf, K. Mullen. Phys. Rev. B 54, 1759-1765 (1996).

128. T. Q. Nguyen, V. Doan, B. J. Schwartz. J. Chem. Phys. 110, $4068-4078$ (1999).

129. T. Q. Nguyen, I. B. Martini, J. Liu, B. J. Schwartz. J. Phys. Chem. B 104, 237-255 (2000).

130. M. Levitus, G. Zepeda, H. Dang, C. Godinez, T. A. V. Khuong, K. Schmieder, M. A. GarciaGaribay. J. Org. Chem. 66, 3188-3195 (2001).

131. D. G. Whitten. Acc. Chem. Res. 26, 502-509 (1993).

132. U. H. F. Bunz. Acc. Chem. Res. 34, 998-1010 (2001).

133. J. S. Yang and T. M. Swager. J. Am. Chem. Soc. 120, 5321-5322 (1998).

134. J. S. Yang and T. M. Swager. J. Am. Chem. Soc. 120, 11864-11873 (1998).

135. M. Halim, J. N. G. Pillow, D. W. Samuel, P. L. Burn. Adv. Mater. 11, 371-374 (1999).

136. S. Son, A. Dodabalapur, A. J. Lovinger, M. E. Galvin. Science 269, 376-378 (1995).

137. N. Johansson, D. A. dosSantos, S. Guo, J. Cornil, M. Fahlman, J. Salbeck, H. Schenk, H. Arwin, J. L. Bredas, W. R. Salanek. J. Chem. Phys. 107, 2542-2549 (1997).

138. N. Johansson, J. Salbeck, J. Bauer, F. Weissortel, P. Broms, A. Andersson, W. R. Salaneck. Adv. Mater. 10, 1136-1141 (1998).

139. F. Steuber, J. Staudigel, M. Stossel, J. Simmerer, A. Winnacker, H. Spreitzer, F. Weissortel, J. Salbeck. Adv. Mater. 12, 130-133 (2000).

140. S. Holdcroft. Adv. Mater. 13, 1753-1765 (2001). 